\title{
Norois
}

Environnement, aménagement, société

$221 \mid 2011$

Innovations et agricultures urbaines durables

\section{Introduction : Innovations et agricultures urbaines durables}

Christophe-Toussaint Soulard, Christine Margetic et Élodie Valette

\section{OpenEdition}

Édition électronique

URL : http://journals.openedition.org/norois/3816

DOI : $10.4000 /$ norois.3816

ISBN : 978-2-7535-1838-4

ISSN : $1760-8546$

Éditeur

Presses universitaires de Rennes

Édition imprimée

Date de publication : 30 décembre 2011

Pagination : 7-10

ISBN : 978-2-7535-1786-8

ISSN : 0029-182X

\section{Référence électronique}

Christophe-Toussaint Soulard, Christine Margetic et Élodie Valette, «Introduction : Innovations et agricultures urbaines durables », Norois [En ligne], 221 | 2011, mis en ligne le 30 décembre 2011, consulté le 19 avril 2019. URL : http://journals.openedition.org/norois/3816 ; DOI : 10.4000/ norois.3816 


\title{
Introduction
}

\section{INNOVATIONS ET AGRICULTURES URBAINES DURABLES}

\author{
Christophe-Toussaint Soulard \\ Géographe, ingénieur de recherche \\ INRA - UMR 0951 INNOVATION \\ 2, place Viala - 34060 MontPelLier cedex 1, France \\ soulard@supagro.inra.fr \\ Christine Margetic \\ Professeure de géographie \\ UMR CNRS 6590 ESO \\ (université de Nantes), \\ Chemin de la Censive-du-Tertre, BP 81227 - 44312 Nantes cedex 3, France \\ christine.margetic@univ-nantes.fr \\ Élodie Valette \\ Géographe, chercheur \\ CIRAD - UMR TETIS \\ Campus International de Baillarguet, TA C-91/F - 34398 MonTPELLier Cedex 5, France \\ elodie.valette@cirad.fr
}

Certains auteurs parlent d'une nouvelle frontière agraire pour qualifier l'enjeu que représente, pour l'alimentation et la santé des populations urbaines de la planète, la reconquête des liens de proximité entre les villes, la nature et l'agriculture (prospective Agrimonde : Paillard et al., 2009). Le concept d'agriculture urbaine résume bien cette idée. Et l'adosser à celui d'innovation n'est pas une surprise car il s'agit a priori d'un oxymore. En effet, penser l'agriculture en ville, ou à partir de la ville, suppose de réviser les conceptions mêmes du fait agricole. La référence aux modèles agricoles généraux, qu'ils soient dominants, tels ceux d'agricultures familiales ou agro-industrielles, ou alternatifs, comme l'agriculture biologique, ne permet pas d'éclairer ce qui se joue dans le renouvellement des liens agricultures-villes.

Dans et autour des villes, différents modèles d'agriculture coexistent, et c'est bien leurs combinaisons territoriales qu'il s'agit de comprendre, au sein de territoires complexes, multifonctionnels et dynamiques. Certains analystes ont proposé des concepts aptes à éclairer cette complexité. En 1997, Bryant proposait de considérer l'agriculture périurbaine comme un milieu innovateur. Cette idée a été développée par M. Poulot (2010) qui identifie les projets agri-urbains comme des lieux d'innovations dès lors qu'ils permettent aux acteurs d'explorer des voies d'articulation entre des intérêts contradictoires et entre des actions sectorielles, dans des territoires en émergence. Ces travaux amènent les géographes à s’intéresser au concept d'innovations territoriales (Giraut, 2009) ou d'inter-territorialité (Vanier, 2007), pour décrire les agencements territoriaux qui se créent et se reconfigurent sans cesse, supposant parfois des innovations organisationnelles, socio-spatiales ou institutionnelles (Sabatier et al., 2007). Les agronomes et économistes agricoles sont aussi force de proposition avec le concept d'agriculture de proximité qui suggère une différenciation des systèmes agricoles sous l'effet des dynamiques territoriales, qu'elles soient urbaines ou rurales (Duvernoy et al., 2005). Les architectes et urbanistes participent à ce mouvement en proposant 
Christophe-Toussaint Soulard, Christine Margetic, Élodie Valette

d'autres concepts, tels ceux d'«edible landscape » (Bhatt et al., 2011) ou de « continuous productive urban landscape » (Viljoen, 2009) qui prônent l'invention de nouvelles formes d'agriculture et de production alimentaire au cœur même de la ville, avec ou sans terre, sur les murs, les toits, etc. Citons enfin les nombreux mouvements associatifs nationaux et internationaux qui émergent pour innover grâce à la mise en réseau d'initiatives d'agriculture urbaine. Il suffit par exemple de lire Urban agriculture magazine ${ }^{1}$ pour constater l'omniprésence de l'innovation comme thème d'échanges d'expériences menées de par le monde.

L'innovation apparaît alors constitutive du concept « d'agriculture urbaine ». Mais de quelles innovations parle-t-on? Dans quelles conditions émergent-elles? Pour quelles finalités de développement?

Ces dernières années, plusieurs colloques ont réuni des chercheurs et des praticiens pour avancer sur ces questions. En 2007, le colloque international de Nanterre a permis d'explorer l'enjeu pour la ville de l'agriculture périurbaine et les innovations que suppose son insertion dans les projets de territoire, les projets dits « agri-urbains » (Vidal et al., 2007). En 2008, l'innovation est au cour du colloque qu'organise l'UMR ADES à Bordeaux sur « Les périphéries urbaines, entre normes et innovations ${ }^{2}$ », mais l'agriculture est peu évoquée. En 2007, puis en 2010, deux colloques tenus à Montréal, dans le cadre des congrès de l'ACFAS, ciblent la réflexion sur la gouvernance de l'agriculture dans les territoires urbains et périurbains (Loudiyi et al., 2008; Bryant et al., 2010). Le symposium «Innovation and Sustainable Development in Agriculture and Food » tenu en 2010 à Montpellier, renouvelle la question à partir d'une double entrée agricole et alimentaire (Coudel et al., 2010). La problématique de l'innovation face aux enjeux du développement durable sert de grille d'organisation des sessions et des contributions. Ce numéro de Norois regroupe des contributions issues de ce symposium ISDA ainsi que deux autres articles ayant permis de structurer un ensemble cohérent. Il explore différentes situations d'innovations pour des agricultures urbaines durables.

Les contributions qui traitent de durabilité agricole mettent l'accent sur les pratiques et les organisations innovantes d'agriculteurs, ou de citadins pratiquant l'agriculture, pour s'adapter à l'environnement urbain. À partir de trois cas d'étude, en périphérie de Paris, Dakar et Antananarivo, Awa Ba et Christine Aubry montrent la complexité des dynamiques agricoles urbaines, ce qui les conduit à élargir les concepts de l'agronomie-système pour analyser les innovations techniques, commerciales, organisationnelles dans les exploitations agricoles périurbaines. En effet, comprendre ces dynamiques suppose d'articuler la double durabilité « interne » et « externe » de l'exploitation agricole, et d'élargir l'analyse du système de production agricole au système d'activités et au système de commercialisation. S'appuyant également sur la démarche comparative, ici les systèmes maraîchers à Buenos Aires et à Stockholm, Camille Hochedez et Julie Le Gall montrent que le développement de ces systèmes au sein de l'espace urbain est porté par des néo-agriculteurs pauvres qui s'organisent en réseaux pour commercialiser leurs produits. Les innovations locales, économiques et sociales, qui émergent de ces organisations d'agriculteurs sont ensuite reprises dans un second temps par les pouvoirs publics qui les insèrent dans des dispositifs législatifs entérinant les recompositions territoriales face aux enjeux fonciers. La gouvernance (Lardon et al., 2008) devient alors une notion-clé de l'application territoriale du concept de développement durable.

Les deux contributions suivantes abordent les questions d'inégalités socio-spatiales en périurbain. À partir de l'étude de l'insertion territoriale du bâti agricole en milieu périurbain, Brigitte Nougarèdes montre comment un nouveau concept d'aménagement, le hameau agricole, réinterroge la dimension sociale de la politique locale de l'habitat : mixité sociale et justice spatiale sont au cœur des négociations locales, au sein du monde agricole, mais aussi dans les relations de voi-

1. Cf. RUAF, site web : [http://www.ruaf.org/node/100]

2. Les périphéries urbaines entre normes et innovations. Les villes du sud de l’Europe, Colloque ADES, Bordeaux, du 11 au 13 juin 2008 
sinage et dans l'action municipale. Hector Ávila-Sanchez analyse les formes d'agriculture présentes dans les périphéries urbaines du centre du Mexique et fait ressortir les inégalités socio-spatiales qui s'expriment, en particulier quant au sort réservé aux terres communes et aux ejidos occupés par des agriculteurs pauvres, menacés d'expropriation. Au-delà de leurs contrastes géographiques, ces deux études avancent la même thèse, celle d'un impensé collectif quant au rôle crucial des sociabilités locales dans l'aménagement durable.

Un paradoxe se fait jour : ces agricultures urbaines, menacées par la croissance urbaine, contribuent pourtant à formuler une réponse aux crises urbaines qu'engendre cette croissance.

Cette ambivalence de l'agriculture périurbaine est illustrée par les deux articles suivants qui traitent de la gouvernance multi-acteurs. Dans la région italienne du Chianti, Coline Perrin retrace l'histoire des relations entre les municipalités et le consorzio des viticulteurs. Les tentatives d'intégration du vignoble du Chianti révèlent deux processus de territorialisation concurrents : celui du terroir viticole, agricole et élitiste, et celui de la proximité urbaine, territoriale et citadine. Dans un contexte plus asymétrique pour l'agriculture, mais avec une issue comparable, José Serrano et Gisèle Vianey décryptent les jeux d'acteurs dans l'aménagement d'une périphérie de l'est tourangeau. Ils montrent le poids déterminant de certains acteurs économiques, ici des transporteurs, dans les choix fonciers de communes pourtant encore agricoles. Dans les deux cas, entre conflits et compromis, l'hybridation entre acteurs porteurs de modèles de développement concurrents explique les régulations foncières observées dans les territoires périurbains.

Concevoir l'espace agricole comme un espace public est-il alors une voie à explorer pour aller dans le sens de sa préservation et de son intégration au système urbain? C'est l'une des idées qu'instillent les nouveaux principes et dispositifs législatifs inclus dans les politiques publiques, qui tous inscrivent le « développement durable »- dans son acception la plus commune, c'est-àdire la plus floue - comme finalité. Les deux derniers textes éclairent ce point. Camille Clément et Patricia Abrantes dressent le paysage des législations et des dispositifs d'intervention publique aux différentes échelles d'action, de l'Europe au local. La comparaison France-Portugal s'avère féconde pour montrer à la fois la convergence des objectifs d'action publique et les décalages entre les actions prescrites et les dynamiques locales. C'est à ce niveau que la traduction des problématiques du développement durable s'opère, les effets de contextes restant néanmoins déterminants. Un zoom plus précis est apporté par le dernier texte de Maud Balestrat et al. qui pose la question centrale du devenir du patrimoine « sol agricole ». Une initiative de cartographie des sols en Languedoc-Roussillon sert de fil conducteur à une réflexion sur le rôle de l'information dans l'orientation vers un aménagement durable.

Complexité des jeux d'acteurs, disparité des contextes tant spatiaux qu'institutionnels, la question de la durabilité des agricultures urbaines s'avère au final une clé d'entrée opportune pour mettre en exergue nombre d'innovations sociales, techniques et commerciales, organisationnelles ou institutionnelles, qui contribuent à redéfinir localement la nature du lien villes-agricultures. Les exemples illustrent cependant l'instabilité et les limites de ces innovations dont le succès peut toujours être remis en cause. Or, l'enjeu est bien de repérer, d'impulser et d'évaluer les combinaisons vertueuses d'innovations au sein des systèmes et des territoires, susceptibles de se traduire en innovations territoriales durables. Cet immense défi n'est pas seulement du côté de l'action, car l'innovation se doit aussi d'être du côté de la recherche. Comme on l'a vu, l'analyse des agricultures urbaines nécessite une révision des concepts et méthodes classiquement utilisés dans la recherche agricole. Inversement, la conception de la durabilité des systèmes urbains s'enrichit de la prise en compte des dynamiques agricoles.

Le présent numéro espère contribuer à ce décloisonnement disciplinaire. Il apporte les pièces d'un puzzle à agencer pour tenter de conceptualiser des systèmes ou des territoires agro-urbains durables. Réalisme ou utopie? Au lecteur de se forger son propre jugement! 


\section{Bibliographie}

Bhatt V., Simopoulos A. P., (dir.), 201 l. Urban Agriculture and Urban Design. Healthy Agriculture, Healthy Nutrition, Healthy People, World Rev Nutr Diet., Basel, Karger, vol. 102, p. 226-243.

Bryant C., 1997. L'agriculture périurbaine : l'économie politique d'un espace innovateur, Cahiers Agricultures, 6, p. 125-130.

Coudel E., Devautour H., Soulard C.-T., Hubert B. (dir.), 2010. Innovation \& Sustainable Development in Agriculture and Food, Symposium proceedings, ISDA, 2010, [http://hal.archives-ouvertes.fr/ISDA2010].

Duvernoy I., Jarrige F., Moustier P., Serrano J., 2005. Une agriculture multifonctionnelle dans le projet urbain : quelle reconnaissance, quelle gouvernance?, Cahiers de la multifonctionnalité, nº 8, p. 87-104.

Giraut, 2009. Innovation et territoires. Revue de géographie alpine, 97-1, [http://rga.revues.org/index783. html/].

Lardon S., Chia E., Rey-Valette H., 2008. Introduction : Dispositifs et outils de gouvernance territoriale, Norois, n 209/4, [http://norois.revues.org/index2602.html].

Loudiy S., Bryant C., Laurens L. (dir.), 2008. Territoires périurbains et gouvernance : perspectives de recherche, $75^{\mathrm{e}}$ Congrès de l'ACFAS, Trois-Rivières, Québec (CAN).

Paillard S., Treyer S., Dorin B. (coord.), 2009. Agrimonde. Scénarios et défis pour nourrir le monde en 2050, Paris, Quae, 295 p.

Poulot M., 2010. L'agriculture comme composante de l'identité périurbaine francilienne : entre (re)connaissance et innovation, Pour, n 205-206: p. 73-81.

Sabatier B., Soulard C., Jarrige F., Laurens L., Nougarèdes B., 2007. L'agriculture périurbaine aujourd'hui : un champ de recherche multidimensionnel, Colloque Les agricultures périurbaines, un enjeu pour la ville. Vers des projets de territoire, Paris, France, [http://halshs.archives-ouvertes. fr/halshs-00348085/fr/].

VAnier M, 2007. Le pouvoir des territoires. Essai sur l'interterritorialité, Paris, Economica, 160 p.

VidAL R., FLEURY A., POULOT M., (coord.) 2007. Les agricultures périurbaines, un enjeu pour la ville. Vers des projets de territoire. Colloque UPX - GECKO, ENSP Versailles, Nanterre, 1-2 octobre 2007.

Viljoen A. (eds.), 2009 (2005). CPULS : continuous productive urban landscapes. Designing agriculture for sustainable cities, Elsevier Ltd/Architectural Press, Oxford (UK). 